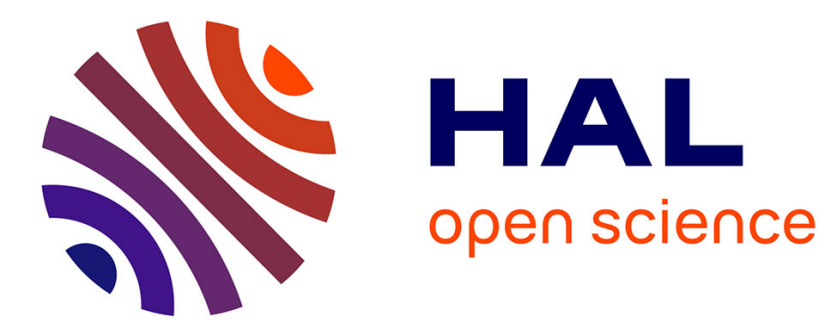

\title{
Magnétorésistance géante dans les nanostructures magnétiques
}

\author{
A. Fert, R. Morel, A. Barthélémy, V. Cros, Jean-Luc Duvail, J.-M. George, F. \\ Parent, F. Petroff, C. Vouille
}

\section{- To cite this version:}

A. Fert, R. Morel, A. Barthélémy, V. Cros, Jean-Luc Duvail, et al.. Magnétorésistance géante dans les nanostructures magnétiques. Journal de Physique IV Proceedings, 1997, 07 (C6), pp.C6-151-C6-161. 10.1051/jp4:1997613 . jpa-00255712

\section{HAL Id: jpa-00255712 https://hal.science/jpa-00255712}

Submitted on 1 Jan 1997

HAL is a multi-disciplinary open access archive for the deposit and dissemination of scientific research documents, whether they are published or not. The documents may come from teaching and research institutions in France or abroad, or from public or private research centers.
L'archive ouverte pluridisciplinaire HAL, est destinée au dépôt et à la diffusion de documents scientifiques de niveau recherche, publiés ou non, émanant des établissements d'enseignement et de recherche français ou étrangers, des laboratoires publics ou privés. 


\title{
Magnétorésistance géante dans les nanostructures magnétiques
}

\author{
A. Fert, R. Morel*, A. Barthélémy, V. Cros, J.-L. Duvail, J.-M. George, F. Parent, F. Petroff \\ et C. Vouille \\ Unité Mixte CNRS-Thomson, Domaine de Corbeville, 91404 Orsay, France \\ * Groupe des Couches Minces, Université de Montréal, Montréal, Québec, Canada
}

\begin{abstract}
Since its discovery in magnetic multilayers, giant magnetoresistance has become one of the main research topics in both appiied and pure magnetism. Their magnetic and transport properties call for new theories, specially to explain the coupling mechanism between layers and the way spin-dependent scattering gives rise to giant magnetoresistance. Also, this class/of new materials calls for study of their structural properties. In this paper, we review some experimental results and theoretical models on GMR in magnetic multilayers. Then, we present an overview of the giant magnetoresistance in slightly different nanostructures - granular alloys and hybrid systems - where magnetic layers are replaced by nanometric clusters.
\end{abstract}

Résumé : Depuis sa découverte dans les multicouches magnétiques, la magnétorésistance géante est un des champs de recherches les plus actifs en magnétisme, tant fondamental qu'appliqué. Au départ, sa compréhension a nécessité la formulation de théories nouvelles expliquant l'oscillation de couplage entre couches et les mécanismes de transport électronique polarisé en spin qui la sous-tendent. De plus, les multicouches magnétiques représentant alors une toute nouvelle classe de matériaux, de nombreuses études s'attardèrent a en élucider les propriétés structurales. Dans cet article, nous passerons en revue différentes théories et résultats expérimentaux ayant trait à la magnétorésistance géante des multicouches magnétiques. Ensuite, nous présenterons un aperçu des propriétés magnétorésistives de matériaux d'un type légèrement différent - les alliages granulaires et les structures hybrides oủ les entités magnétiques sont présentes sous forme d'agrégats.

\section{INTRODUCTION}

Les multicouches magnétiques constituent un type nouveau de nanostructures, intéressantes de par leurs propriétés électroniques et magnétiques inusitées. Elles sont obtenues par dépôt successif, sur un substrat, de couches magnétiques, par exemple $\mathrm{Fe}$ ou $\mathrm{Co}$, et de couches non magnétiques, par exemple $\mathrm{Cr}, \mathrm{Ag}$ ou $\mathrm{Cu}$. Les épaisseurs des couches individuelles varient de $5 \AA$ à $100 \AA$, soit quelques plans atomiques. Une des premières manifestations des propriétés nouvelles de ces structures fut l'observation, en 1986, d'un couplage antiferromagnétique entre couches de fer dans des tricouches $\mathrm{Fe} / \mathrm{Cr} / \mathrm{Fe}$ déposées par épitaxie par jets moléculaires sur AsGa [1]. Ce couplage antiferromagnétique entraîne, en champ nul, une configuration antiparallèle des aimantations des couches magnétiques successives. L'origine de ce couplage antiferromagnétique, observé depuis dans de nombreuses structures magnétiques en multicouches. repose sur un couplage d'échange indirect, similaire au couplage RKKY présent dans les alliages magnétiques dilués [2]. Qui plus est, le couplage entre couches magnétiques présente un caractère oscillant, alternant entre couplage ferromagnétique $(F)$ et couplage antiferromagnétique (AF) en fonction de l'épaisseur de la couche intercalaire. Ces oscillations, dont la période dépend de la topologie de la surface de Fermi du métal intercalaire, sont elles aussi bien décrites par les modèles de type RKKY [3]. 
Dans ce papier, notre attention se portera particulièrement sur la magnétorésistance géante (GMR), qui pour sa part fut découverte en 1988 dans des multicouches $\mathrm{Fe} / \mathrm{Cr}$ [4]. La GMR se manifeste par une chute prononcée de la résistivité électrique lorsque, sous l'effet d'un champ magnétique externe, les aimantations de couches soumises à un couplage $\mathrm{AF}$ sont progressivement alignées selon une direction commune. Après une description générale de cet effet, nous discuterons les différents modèles théoriques qui ont été proposés pour en définir et en expliquer les mécanismes. Nous présenterons ensuite quelques caractéristiques de nanostructures différentes mais présentant des propriétés similaires : les alliages de type granulaire, où des agrégats magnétiques sont noyés dans une matrice conductrice, et les structures hybrides, contenant à la fois des couches minces et des agrégats magnétiques.

\section{MAGNÉTORÉSISTANCE DES MULTICOUCHES MAGNÉTIQUES}

\subsection{Manifestations expérimentales}

On peut voir à la figure 1 les courbes d'aimantation et de magnétorésistance de multicouches (Co $15 \AA / \mathrm{Cu} 9 \AA)_{15}$ déposées par pulvérisation cathodique. La courbe d'aimantation en fonction du champ appliqué est typique de celles observées dans des multicouches où le couplage est antiferromagnétique. On observe une rémanence faible et un fort champ de saturation. Le champ de saturation étant une mesure du couplage, nous pouvons déduire que dans ce cas-ci, avec $9 \AA$ de cuivre; l'intensité du couplage $J$ est de $-0,24 \mathrm{erg} / \mathrm{cm}^{2}$ [5]. Sur la même figure, on peut voir l'évolution de la résistivité en fonction du champ appliqué. Lorsque celui-ci est suffisant pour vaincre le couplage indirect, la résistivité diminue : c'est l'effet de magnétorésistance géante. Le plus généralement, la GMR est définie par la variation relative de résistivité $\rho$ par rapport à sa valeur minimale à la saturation, soit GMR $=\left(\rho_{\max }-\rho_{\min }\right) / \rho_{\min }$. Dans l'exemple présenté, la GMR mesurée est de $78 \%$ à $4,2 \mathrm{~K}$ et de $45 \%$ à la température ambiante. La magnétorésistance est attribuée à la diffusion dépendante du spin et à la possibilité, sous champ appliqué, de modifier l'orientation relative des aimantations des couches successives, les faisant passer d'une configuration antiferromagnétique a une configuration ferromagnétique. L'arrangement antiferromagnétique des aimantations peut résulter d'un couplage d'échange, tel que décrit plus haut, ou d'une différence de champ coercitif entre couches successives [6], ou encore, dans une tricouche, de l'ancrage d'une des deux couches magnétique [7].
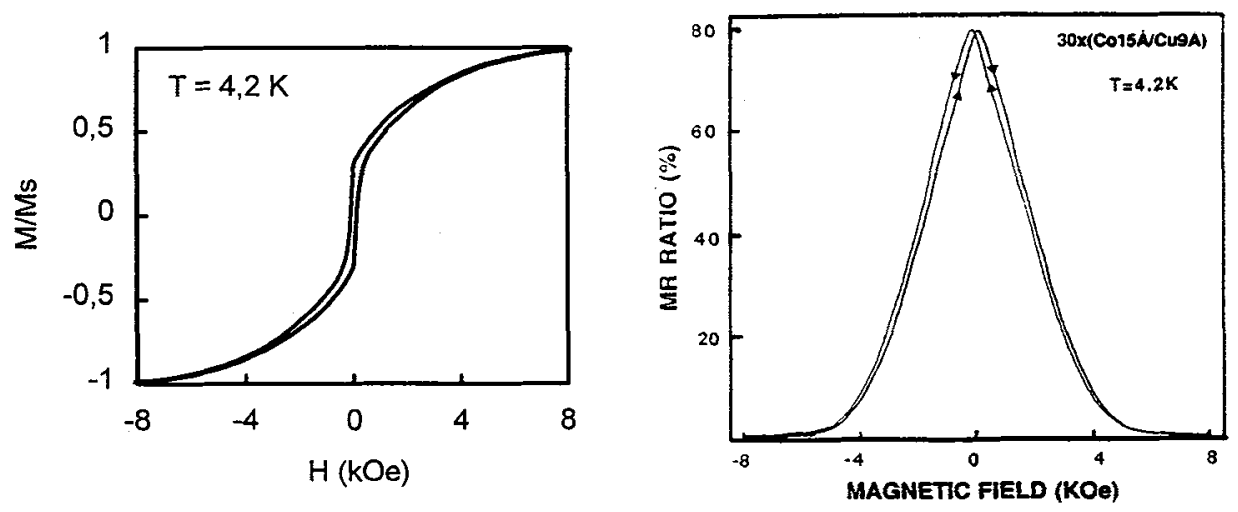

Figure 1 : Aimantation (à gauche) et magnétorésistance géante (à droite) de multicouches (Co $15 \AA / C u 9 \AA$ ) 15 . Les mesures sont effectuées à $4,2 \mathrm{~K}$. 


\subsection{Origine et mécanismes}

De façon phénoménologique, la magnétorésistance repose sur un modèle où le courant électrique, dans un ferromagnétique à basses température, est porté par deux canaux parallèles d'électrons de spins différents (up et down) [8]. Dans certaines conditions, on observe une forte différence entre la résistivité $\rho_{\uparrow}$ d'électrons dont la direction de spin est parallèle à la direction de l'aimantation et la résistivité $\rho_{\downarrow}$ d'électrons dont la direction de spins est antiparallèle à la direction de l'aimantation. Cette différence s'exprime par le rapport $\alpha$ des résistivités, $\alpha=\rho_{\downarrow} \rho_{\uparrow}$. Pour un champ appliqué supérieur au champ de saturation de la multicouche, toutes les aimantations sont colinéaires. C'est donc dire qu'un électron de spin donné, traversant d'une couche magnétique à l'autre, circule toujours dans un canal de même résistivité. Dans le cas où $\alpha$ est très différent de l'unité, on aura alors un effet de court-circuit à travers le canal de plus faible résistivité. (A la figure 2 , on a choisi $\alpha>1$, d'où un court circuit à travers le canal $\rho \uparrow$, et $\rho_{\min }=\rho_{\uparrow}$.) A l'opposé, quand les aimantations des couches successives sont dans une configuration antiferromagnétique, la résistivité pour un canal de spin donné passera alternativement de $\rho_{\uparrow}$ à $\rho_{\downarrow}$ (ou l'inverse), selon l'orientation des spins par rapport à la direction de l'aimantation de la couche traversée. La résistivité dans chaque canal sera alors une moyenne entre $\rho_{\uparrow}$ et $\rho_{\downarrow}$, avec une résistivité globale supérieure à la résistivité en configuration parallèle, $\rho_{\max }=\left(\rho_{\uparrow}+\rho_{\downarrow}\right) / 2$. La chute de résistivité lors de l'application d'un champ magnétique reflète donc le passage d'une configuration parallèle à une configuration antiparallèle des aimantations. D'autre part, pour que ce mécanisme soit efficace, il faut que le libre parcours moyen de l'électron soit supérieur à l'épaisseur des couches, sans quoi l'effet vanne de spin que nous venons de décrire ne peut se manifester. On observe dans ce cas un découplage des processus de diffusions électroniques entre les couches et une diminution graduelle de la GMR.
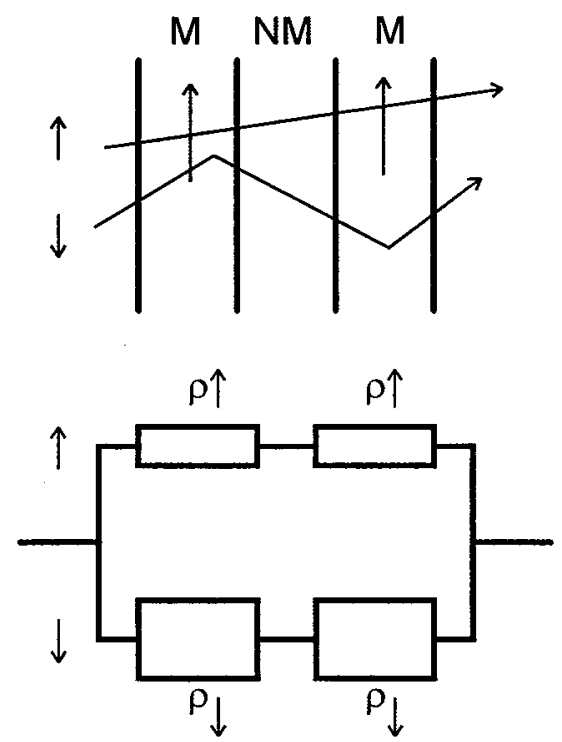
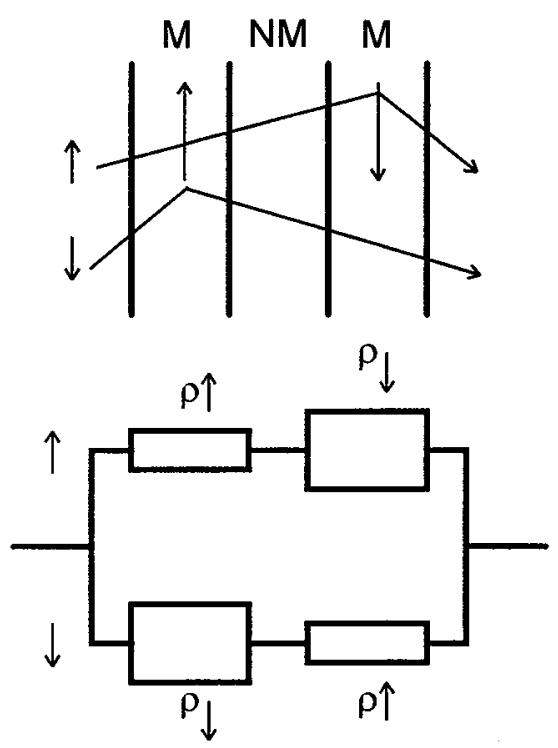

Figure 2: Schéma de principe de la magnétorésistance géante dans une multicouche. A gauche, avec une configuration ferromagnétique des aimantations et, à droite, avec une configuratión antiferromagnétique. Les figures du haut illustrent la trajectoire des électrons, plus fortement diffusés quand leur direction de spin est antiparallèle à la direction locale de l'aimantation. Les figures du bas illustrent la représentation équivalente à l'aide du modèle à deux courants, où les résistivités propres à chaque canal varient selon la configuration des aimantations. 


\subsection{Modèles théoriques}

L'approche semi-classique fut au départ formulée par Camley et Barnas [9]. Il s'agit d'une extension aux multicouches du modèle Fuchs-Sondheimer, décrivant les propriétés de transport électronique des films minces [10]. La diffusion dans le volume et aux interfaces est, dans cette approche, décrite de façon phénoménologique par l'introduction de libres parcours moyens dépendants du spin, et de conditions aux frontières où les coefficients de transmission d'une couche à l'autre dépendent aussi du spin. Outre le traitement numérique du problème, dû à Camley et Barnas [9] et Trigui [11], une solution analytique approchée a été formulée par Barthélémy et Fert [12].

Plusieurs améliorations ont été apportées à la formulation originale de Camley et Barnas : Johnson et Camley ont par exemple décrit l'interface comme une couche en soi, caractérisée par une épaisseur finie et un libre parcours moyen dépendant du spin [13]. Hood et al. [14] introduisirent des potentiels dépendant du spin aux interfaces, de façon à prendre en compte la différence de population entre les deux directions de spins. Dieny [15] introduisit pour sa part une anisotropie des libres parcours moyens, prenant ainsi en compte la structure granulaire des alliages pulvérisés. Finalement, toujours dans le cadre d'un modèle semi-classique, Duvail et al. [16] s'intéressèrent à la dépendance en fonction de la température et de l'épaisseur de la résistivité et de la magnétorésistance de multicouches $\mathrm{Co} / \mathrm{Cu}$.

Ces modèles semi-classique décrivent correctement la magnétorésistance géante, ainsi que sa dépendance en fonction de la température et des épaisseurs des couches $[12,16]$. Cependant, lorsque la diffusion aux interfaces domine, il est en général difficile de décrire la résistivité et la GMR avec un même ensemble de paramètres. Dans le cas de modèles quantiques, on traite la diffusion des électrons par des potentiels dépendant du spin aux interfaces et en volume [17,18]. Les deux processus sont traités sur un pied d'égalité, et les calculs de résistivité et de GMR sont plus cohérents.

\subsection{Caractéristiques principales}

Dans les multicouches, la magnétorésistance géante dépend pour beaucoup du couplage entre couches magnétiques. Ce dernier oscille entre couplage ferromagnétique $(F)$ et couplage antiferromagnétique (AF) selon l'épaisseur de la couche intercalaire. La GMR est maximale quand le couplage est de type $\mathrm{AF}$, et nulle quand le couplage est de type $\mathrm{F}$. A ce comportement oscillant de la GMR s'ajoute une décroissance en fonction de l'épaisseur des couches, témoignant du découplage progressif des processus de diffusion d'une couche à l'autre. La figure 3, qui présente la variation de la GMR en fonction de l'épaisseur de cuivre dans des multicouches $\mathrm{Co} / \mathrm{Cu}$, illustre ces phénomènes. Le premier pic, à $9 \AA$, correspond à une multicouche où le couplage antiferromagnétique est maximum. D'autre part, l'extinction de la GMR entre $12 \AA$ et $15 \AA$ indique qu'à ces épaisseurs, le couplage entre couches de cobalt est ferromagnétique : Lors de l'application d'un champ magnétique, les aimantations des couches tournent à l'unisson sans qu'il y ait modification de leur orientation relative. A plus grandes épaisseurs, la diminution d'amplitude des maximums résulte d'un affaiblissement progressif du couplage $\mathrm{AF}$, ainsi que d'une diminution de la corrélation entre les processus de diffusion d'une couche à l'autre (les épaisseurs de couches devenant comparables aux libres parcours moyens électroniques).

Pour bien comprendre les mécanismes de la magnétorésistance géante, il est nécessaire de connaître la nature et la position (aux interfaces ou en volume) des potentiels diffuseurs dont elle tire son origine. A cette fin. il est intéressant d'étudier l'évolution de la GMR quand une couche, très fine, d'un élément étranger est insérée entre les couches magnétiques et non magnétiques d'une multicouche. De telles expériences ont été effectuées dans les systèmes $\mathrm{Fe} / \mathrm{Cr}$ [19], $\mathrm{NiFe} / \mathrm{Cu}[20,21]$, et $\mathrm{Co} / \mathrm{Cu}$ [22]. $\mathrm{Par}$ exemple, l'insertion de $4,4 \AA$ de Co aux interfaces de multicouches $\mathrm{NiFe} / \mathrm{Cu}$ entraîne une augmentation de la GMR, qui passe de $10 \%$ à $40 \%$. La couche de cobalt étant trop mince pour que l'on puisse 
considérer des effets de diffusion en volume, on conclut de ce résultat que, du moins dans les systèmes $\mathrm{Co} / \mathrm{Cu}$, la contribution des diffusions aux interfaces domine les effets de magnétorésistance géante. Cette observation est d'ailleurs confirmée par la baisse de GMR enregistrée lorsque, dans une expérience complémentaire à celle décrite, des couches minces de fer sont introduites aux interfaces de multicouches $\mathrm{Co} / \mathrm{Cu}$. De plus, en étudiant le comportement en fonction de l'épaisseur de cobalt dans ces multicouches $\mathrm{Co} / \mathrm{Cu}$ dont on a neutralisé les interfaces par l'introduction de couches de fer, George et al. [22] ont mis en évidence une contribution, faible mais non négligeable, de la diffusion en volume dans le cobalt.

Puisque la magnétorésistance géante est, dans bien des cas, dominée par les processus diffusifs aux interfaces, elle sera très sensible aux conditions de dépôt et de croissance de la multicouche, dans la mesure où ceux-ci influencent la rugosité d'interface. En caractérisant la rugosité d'interface, par des mesures magnétiques et de diffraction $\mathrm{X}$, de multicouches $\mathrm{Fe} / \mathrm{Cr}$ pulvérisées dans différentes conditions, Fullerton et al. concluent que dans ces systèmes une augmentation de la rugosité augmente la GMR [24]. D'autre part, pour le même système $\mathrm{Fe} / \mathrm{Cr}$, Petroff et al. observent une augmentation de la GMR pour des recuits à des températures inférieures à $300^{\circ} \mathrm{C}$ (ayant pour conséquence une augmentation de la rugosité) alors que des températures de recuit supérieures ont pour effet de diminuer la GMR. Ces résultats laissent supposer qu'il existe un degré optimum de rugosité où la magnétorésistance est maximale.

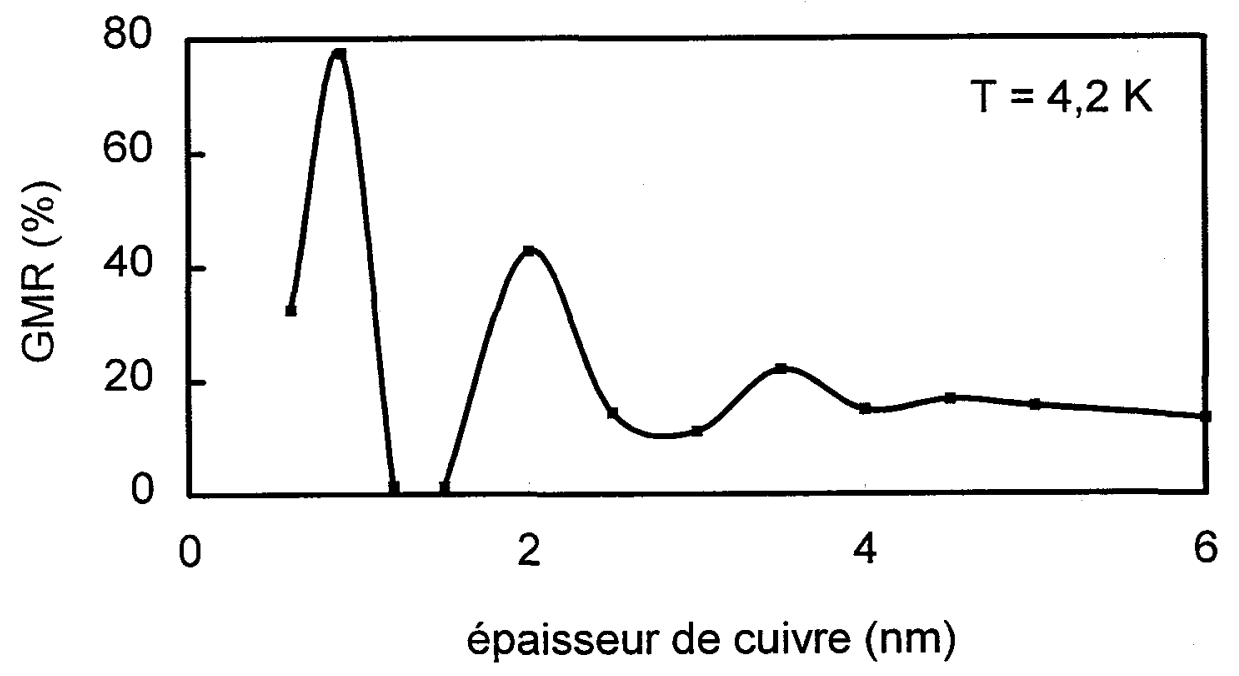

Figure 3 : Variation de la magnétorésistance dans des multicouches $\mathrm{Co} / \mathrm{Cu}$, en fonction de l'épaisseur de cuivre. L'épaisseur de cobalt est de $15 \AA$; les mesures sont effectuées à $4,2 \mathrm{~K}$.

La magnétorésistance géante présente aussi une variation en fonction de la température : Lorsque celle-ci augmente, les diffusions électron-magnon induisent un renversement de spin de l'électron, dont la conséquence est un mélange graduel des deux canaux de spins. Suite à ce mélange, la résistivité devient peu à peu égale dans les deux canaux, et la GMR diminue. Toutefois, la diminution en fonction de la tempérarure n'est pas la même dans tous les systèmes. Elle est relativement faible dans les multicouches $\mathrm{Co} / \mathrm{Cu}$, et plus forte dans les systèmes $\mathrm{Fe} / \mathrm{Cr}$ ou $\mathrm{Fe} / \mathrm{Cu}[16]$. 
La dernière caractéristique de la magnétorésistance géante que nous voudrons aborder concerne la géométrie même de la mesure. De façon naturelle - en déposant des contacts électriques sur la surface de l'échantillon - le courant injecté dans la multicouche circule dans le plan des couches. C'est la géométrie dite CIP (Current In Plane). Cependant, des effets de GMR encore plus importants que ceux observés en géométrie CIP apparaissent quand l'injection de courant est faite perpendiculairement au plan des couches. Cette géométrie, dite CPP (Current Perpendicular to the Plane), est beaucoup plus difficile à mettre en oeuvre car elle implique soit l'utilisation de contacts supraconducteurs, pour minimiser les résistances de contact, soit la découpe par lithographie de petits éléments de multicouches, pour augmenter jusqu'à un niveau mesurable la résistance de l'échantillon.

L'augmentation de la magnétorésistance mesurée en configuration CPP est attribuée à deux facteurs.

Puisque dans ce cas le transport électronique se fait à travers les couches successives, il est normal que les effets de diffusion aux interfaces soient accentués. D'autre part, il y a en CPP accumulation de spins aux interfaces en raison de différences de résistivité entre canaux de spins. Cet effet, absent en configuration CIP, accentue l'effet des différences entre canaux. La formulation même de la théorie de la GMR est d'ailleurs modifiée en CPP pour tenir compte de cet effet et présente l'avantage de bien séparer les deux contributions de volume et d'interfaces [27]. Il est alors possible de déterminer sans ambiguité la valeur des coefficients $\alpha$ (le rapport des résistivités des deux canaux de spins) pour l'interface et le volume. Par exemple, dans des multicouches $\mathrm{Co} / \mathrm{Cu}$ on trouve $\alpha=7,5$ aux interfaces et $\alpha=3$ dans le volume [27], confirmant ainsi la contribution prépondérante de la diffusion dépendant du spin aux interfaces.

\section{AUTRES NANOSTRUCTURES MAGNÉTORÉSISTIVES}

\subsection{Magnétorésistance géante d'alliages granulaires}

Outre les multicouches, d'autres types de nanostructures incluant métal magnétique et métal non magnétique présentent des effets de magnétorésistance géante. Par exemple, Berkowitz et al. [29] et Xiao et al. [30] mirent en évidence une forte GMR dans des alliages granulaires obtenus par dépôt simultané d'un couple de métaux non miscibles, tels cobalt et argent. Dans ces systèmes, les conditions thermodynamiques hors équilibre qui prévalent lors de la co-déposition conduisent à la formation de précipités ferromagnétiques de tailles nanométriques dans une matrice conductrice. En champ nul, les aimantations des agrégats magnétiques sont désordonnées, alors que l'application d'un champ magnétique permet de saturer l'aimantation dans une direction donnée. Pour un électron de conduction, cette réorientation des aimantations d'agrégats voisins est similaire à la rotation des aimantations dans une multicouche et engendre une magnétorésistance géante. Par exemple, la GMR atteint, à 4,2 K, 75\% dans des alliages pulvérisés $\mathrm{Co}_{20} \mathrm{Ag}_{80}$ [31], $50 \%$ dans des alliages $\mathrm{Co}_{25} \mathrm{Cu}_{75}$ obtenus par épitaxie par jets moléculaires [32], et $43 \%$ dans des alliages $\mathrm{Fe}_{20} \mathrm{Ag}_{80}$ [33]. De même, certains alliages ternaires ont été étudiés et on trouve une GMR de $47 \%$ dans des granulaires $\left(\mathrm{Ni}_{80} \mathrm{Fe}_{20}\right)_{20} \mathrm{Ag}_{80}$ pulvérisés [34] ou encore $75 \%$ dans des alliages $\left(\mathrm{Fe}_{30} \mathrm{Co}_{70}\right)_{36} \mathrm{Cu}_{64}$ obtenus par trempe sur roue [35]. Ces résultats sont comparables aux magnétorésistances obtenues dans des multicouches de compositions similaires.

En fonction de la concentration relative des constituants, la GMR des alliages granulaires présente, de façon générale, un maximum quand la concentration atomique du constituant magnétique est de l'ordre de $30 \%[33,36]$. Au-delà de cette concentration, il y a percolation des agrégats magnétiques et donc perte du désordre d'orientation à champ nul sans lequel il n'y a pas de magnétorésistance géante. Aux basses concentrations, il est naturel de supposer que les centres de diffusion dépendants du spin - qu'ils soient en volume ou aux interfaces - ne sont pas assez nombreux pour engendrer une forte dépendance en spin de la résistivité. 
Au-delà de ces considérations qualitatives, l'énoncé d'une théorie de la GMR dans les alliages granulaires doit prendre en compte aussi bien les mécanismes de la diffusion des électrons en milieu non homogène, que les propriétés magnétiques des petites particules, qui sont très différentes de celles des ferromagnétiques massifs. De tels modèles ont été énoncés, par exemple par Zhang et Levy, dans le cadre d'un formalisme de Kubo [37]. Les prédictions de ce modèle - en ce qui concerne la dépendance de la GMR en fonction de la taille et de la concentration des particules - ont été confirmées dans des alliages granulaires $\mathrm{CoCu}[38]$.

Une autre caractéristique de la magnétorésistance des alliages granulaires est sa dépendance en fonction du champ magnétique appliqué : A mesure que la taille des agrégats est réduite, les barrières d'énergie entre directions de facile aimantation diminue. Pour des agrégats dont la taille est inférieure à quelques nanomètres, l'énergie thermique à la température ambiante - ou même en dessous - peut être suffisante pour vaincre l'énergie d'anisotropie. Le système se comporte alors comme un ensemble de particules paramagnétiques, dont le moment serait égal au moment magnétique total de l'agrégat, d'où l'appellation de superparamagnétique. Ce régime est, entre autres choses, caractérisé par une rémanence nulle de l'aimantation et un champ de saturation bien supérieur à celui des ferromagnétiques massifs. La dépendance en champ de la GMR étant le reflet de la dépendance en champ de l'aimantation, elle aussi présentera une saturation beaucoup plus lente que, par exemple, celle des multicouches. Un exemple de magnétorésistance géante en régime superparamagnétique est donné à la figure 4 , où est présentée la GMR d'une couche ultra mince d'alliage granulaire $\mathrm{Co}_{20} \mathrm{Ag}_{80}$. En ajustant la magnétorésistance en fonction du champ à l'aide d'une fonction de Langevin [39], propre à ce régime, on peut évaluer la taille des agrégats magnétiques, dans ce cas-ci 1,6 $\mathrm{nm}$ [40].

Ces deux effets combinés - la dépendance en fonction de la taille et de la concentration de la GMR et le superparamagnétisme des petites particules - expliquent en partie la variation de la GMR en fonction de la structure de l'alliage. Par exemple, celle-ci est très sensible aux effets de recuits thermiques. Au départ, le recuit favorise la croissance d'agrégats par la formation de précipités à partir d'atomes isolés en suspension dans la matrice et réduit la résistivité de l'alliage en éliminant certains défauts de structure.

Ces deux effets concourent à une augmentation de la GMR. D'autre part, les recuits à haute température - au-delà de $300^{\circ} \mathrm{C}$ ou $400^{\circ} \mathrm{C}$ - provoquent la fusion d'agrégats voisins, ce qui tend à réduire le rapport surface/volume et réduit la GMR (là où elle est dominée par les effets de diffusion aux interfaces agrégat/matrice). Des effets similaires d'augmentation de la taille sont obtenus par des dépôts à chaud et conduisent aussi à une augmentation de la magnétorésistance géante.

Cependant, la pulvérisation cathodique, pas plus que l'épitaxie par jets moléculaires, ne permettent un contrôle direct et simultané de la taille et de la distribution des particules. Cette conclusion s'applique tout aussi bien aux techniques alternatives de trempe sur roue [42] ou de broyage mécanique [42,43] utilisées pour la fabrication d'alliages granulaires.

\subsection{Magnétorésistance géante de structures hybrides}

Dès la découverte de la magnétorésistance géante, il apparut que les matériaux à forte GMR pouvaient avantageusement être utilisés pour la détection ou la mesure de champs magnétiques, à condition toutefois d'en augmenter la sensibilité en diminuant leur champ de saturation. Dans les multicouches, le champ de saturation est proportionnel à l'énergie de couplage entre couches magnétiques. Là où le couplage d'échange indirect est la source de cette interaction, il est possible de la réduire à peu de choses puisqu'elle oscille en fonction de l'épaisseur de métal intercalaire : Il s'agit alors de choisir une épaisseur telle que l'interaction soit faible ou nulle. Par contre, même dans de tels cas, il est pratiquement impossible d'éviter les couplages directs entre couches magnétiques par contact à travers des piqûres ou des défauts - toujours présents - dans la couche intercalaire. 


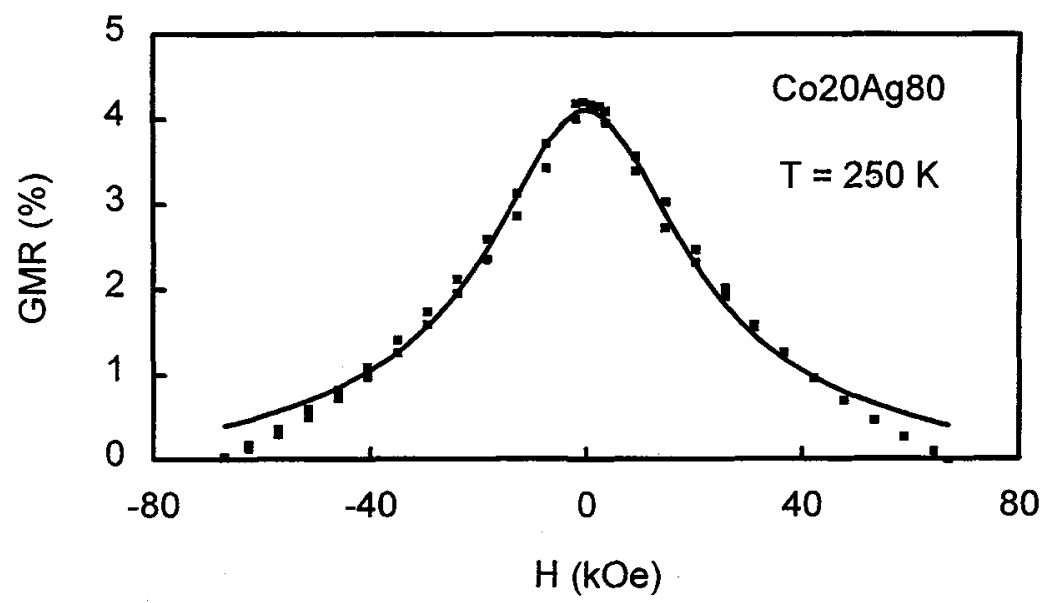

Figure 4 : Magnétorésistance géante dans une couche mince granulaire $\mathrm{Co}_{20} \mathrm{Ag}_{80}$. La courbe en trait plein est calculée, en supposant des agrégats de 1,6 nm de diamètre, à l'aide d'une fonction de Langevin.

Une structure très particulière, permettant justement de contrecarrer l'effet des couplages directs, fut obtenue par Hylton et al. [44] par recuit de multicouches $\mathrm{Ni}_{80} \mathrm{Fe}_{20} / \mathrm{Ag}$. Lors du traitement thermique la diffusion de l'argent le long des joints de grains, qui traversent la multicouche, provoque la rupture en îlots des couches de permalloy $\left(\mathrm{Ni}_{80} \mathrm{Fe}_{20}\right)$. Parce que ces îlots sont découplés, l'ensemble de la structure est moins sensible aux effets de couplage direct et on enregistre des sensibilités de l'ordre de $0,8 \% / \mathrm{Oe}$.

Des structures un peu semblables, avec discontinuité des couches magnétiques, peuvent être obtenues directement par le dépôt de couches magnétiques très minces. Avec certains couples d'éléments où le mouillage lors du dépôt est mauvais $(\mathrm{Co} / \mathrm{Ag}, \mathrm{Co} / \mathrm{Cu})$, on obtient, pour des épaisseurs en dessous de $10 \AA$, des couches magnétiques en deçà du seuil de percolation et donc incomplètes (sinon granulaires). L'effet sur la magnétorésistance géante est immédiat. Dans des multicouches $\mathrm{Co} / \mathrm{Ag}$, avec $15 \AA$ d'argent, la GMR qui est de $10 \%$ avec des couches de cobalt complètes passe à $65 \%$ quand les couches de cobalt sont incomplètes $(4 \AA)$.

Le gain de sensibilité obtenu par l'utilisation de couches granulaires incomplètes est toutefois beaucoup plus évident lorsque celles-ci sont insérées dans des structures - dites hybrides - où alternent couche granulaire de cobalt, couche intercalaire d'argent, et couche complète de permalloy [46]. Le découplage effectif des aimantations du cobalt et du permalloy permettent d'exploiter au maximum le contraste entre leurs coercivités. Comme le montre la figure $5 \mathrm{a}$, le cycle d'hystérésis de la multicouche hybride présente un retournement de l'aimantation en deux temps : Un premier saut à très faible champ ( $2 \mathrm{Oe}-3 \mathrm{Oe})$ correspond au retournement rapide du permalloy, alors que la portion à plus fort champ coercitif (1000 Oe) correspond à la rotation des couches granulaires de cobalt. Vu l'absence, à toutes fins pratiques, de couplage entre les aimantations du cobalt et du permalloy, une configuration antiferromagnétique quasi parfaite résulte de la rotation à bas champ du permalloy (le cobalt étant alors fixe). Ce saut d'aimantation s'accompagne d'une brusque variation de la résistivité, avec une sensibilité à $4,2 \mathrm{~K}$ atteignant $7 \% / O e$. Toutefois, la taille des agrégats de cobalt étant telle que leur température de blocage soit inférieure à la température ambiante, ce comportement n'est observé qu'à basse température. Lorsque les couches granulaires ont un comportement superparamagnétique, leur désordre thermique fait en sorte que le 
retournement des couches de permalloy est sans effet sur l'orientation relative des couches, et la magnétorésistance géante est réduite de façon significative.

Pour que ces structures hybrides trouvent une application pratique, il est donc nécessaire d'augmenter la température de blocage des agrégats. Celle-ci étant fonction, entre autres, de la taille, l'essentiel des efforts en ce sens portent.sur l'étude des conditions de croissance et de dépôt des agrégats de cobalt sur l'argent ou le cuivre.
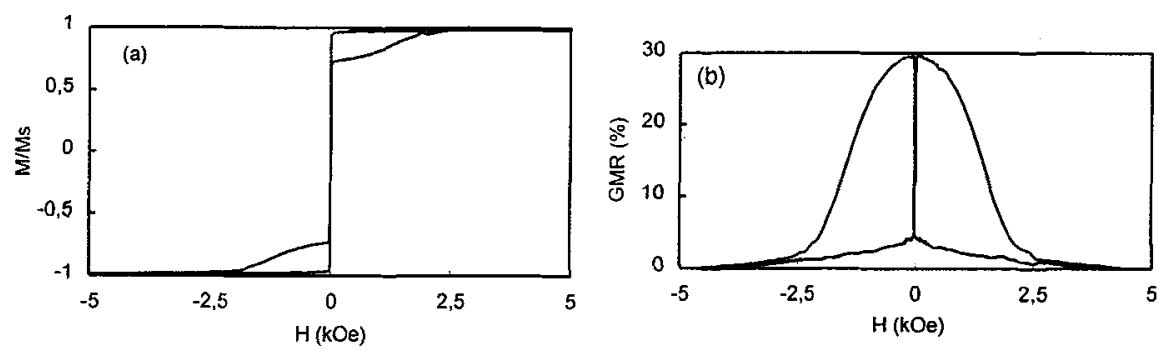

Figure 5 : Aimantation (a) et magnétorésistance géante (b) en fonction du champ magnétique, à $4,2 \mathrm{~K}$, dans une multicouche hybride (Co $4 \AA / \mathrm{Ag} 40 \AA / \mathrm{NiFe} 40 \AA / \mathrm{Ag} 40 \AA)_{15}$.

\section{CONCLUSIONS}

La magnétorésistance géante des multicouches n'est que le premier exemple d'effet mettant en jeu le caractère polarisé du transport électroniques des métaux ferromagnétiques. Comme on l'a vu, des systèmes comme les alliages granulaires où les systèmes hybrides présentent eux aussi des propriétés de transport intéressantes. Les fortes variations de résistivité en fonction du champ magnétique de ces structures ont déjà permis la mise au point de senseurs magnétiques et de têtes de lecture magnétiques plus sensibles que celles utilisées jusqu'alors. De fait, ces nouveaux matériaux sont des candidats sérieux dans la course à la miniaturisation et à l'augmentation de la densité d'information des supports de stockage informatiques. D'autre part, les effets de polarisation en spin du transport électronique peuvent aussi conduire à d'autres réalisations, par exemple les transistor de spins [47], ou encore les dispositifs à injection de spins [48]. La plupart de ces structures nouvelles reposent sur un mariage d'éléments magnétiques, de semi-conducteurs et de matériaux isolants, le plus souvent à l'échelle nanométrique. On comprendra alors que, malgré les efforts de recherches soutenus, il s'agit là d'un champ de recherche où les limites sont imposées beaucoup plus par les techniques de croissance et d'élaboration des nanostructures, que par la physique ou l'imagination des chercheurs.

\section{Références}

[1] Grünberg P., Schreiber R., Pang Y., Brodsky M.B., eţ Sowers H., Phys. Rev. Lett. 57 (1986) 2442.

[2] Parkin S.S.P., More N., et Roche K.P., Phys. Rev. Lett. 64 (1990) 2304.

[3] Bruno P. et Chappert C., Phys. Rev. B 46 (1992) 261.

[4] Baibich M.N., Broto J.M., Fert A., Nguyen Van Dau F., Petroff F., Etienne P., Creuzet G., 
Friederich A., et Chazelas J., Phys. Rev. Lett. 61 (1988) 2472.

[5] Mosca D.H., Petroff F., Fert A., Schroeder P.A., Pratt W.P., et Loloee R., J. Magn. Mag. Mater. 94 (1991) L1.

[6] Dupas C., Beauvillain P., Chappert C., Renard J.P., Trigui F., Veillet P., Velu E., et Renard D J. Appl. Phys. 67 (1990) 5680.

[7] Dieny B., Speriosu V.S., Parkin S.S.P., et Gurney B.A., Phys. Rev. B 43 (1991) 1297.

[8] Fert A. et Campbell I.A., J. Phys. F 6 (1976) 849; Campbell I.A. et Fert A., Ferromagnet Materials (Wohlfarth E.P. éditeur, North-Holland, Amsterdam, 1982) p. 769.

[9] Camley R.E. et Barnas J., Phys. Rev. Lett. 63 (1989) 664.

[10] Fuchs F., Proc. Camb. Phil. Soc. 34 (1938) 100.

[11] Trigui F., Velu E., et Dupas C., J. Magn. Magn. Mater. 93 (1991) 421. Barthélémy A. et Fert A., Phys. Rev. B 43 (1991) 13124. Johnson B.L. et Camley R.E., Phys. Rev. B 44 (1991) 9997.

[15] Dieny B., Europhys. Lett. 17 (1992) 261

[16] Duvail J.L., Fert A., Pereira L.G., et Lottis D.K., J. Appl. Phys. 75 (1994) 7070.

[17] Levy P.M., Zhang S., et Fert A., Phys. Rev. Lett. 65 (1990) 1643; Zhang S., Levy P.M., । Fert A., Phys. Rev. B 45 (1992) 8689.

[18] Vedyayev A., Dieny B., et Ryzhanova N., Europhys. Lett. 19 (1992) 329.

[19] Baumgart P.A., Gurney B.A., Wilhuit D.R., Nguyen T., Dieny B., et Speriosu V.S., J. App Phys. 69 (1991) 4792.

[20] Parkin S.S.P., Phys. Rev. Lett. 71 (1993) 1641.

[21] Dieny B., Speriosu V.S., Nozieres J.P., Gurney B.A., Vedyayev A., Ryzhanova N., Magnetisr and Structure in Systems of Reduced Dimension (Farrow R.F.C. et al. éditeurs, Plenum Pres: New York, 1993) p. 279.

[22] George J.M., Barthélémy A., Petroff F., Valet T., et Fert A., Mat. Res. Symp. Proc. 313 (199Z 737.

[23] Petroff F., Barthélémy A., Mosca D.H., Lottis D.K., Fert A., Schroeder P.A., Pratt W.P Loloee R., et Lequien S., Phys. Rev. B 44 (1991) 5355.

[24] Fullerton E.E., Kelly D.M., Guimpel J., Schuller I.K., et Bruynseraede Y., Phys. Rev. Lett. 6 (1992) 859.

[25] Petroff F., Barthélémy A., Fert A., Etienne P., et Lequien S., J. Magn. Magn. Mater. 93 (1991 95.

[26] Schroeder P.A., Magnetism and Structure in Systems of Reduced Dimension (Farrow R.F.C. 6 al. éditeurs, Plenum Press, New York 1993) p. 129.

[27] Fert A. et Valet T., J. Magn. Magn. Mater. 121 (1993) 378; Valet T. et Fert A., Phys. Rev. B 4 (1993) 7099.

[28] Lee S.F., Pratt W.P., Yang Q., Holody P., Loloee R., Schroeder P.A., et Bass J., J. Magr Magn. Mater. 118 (1993) L1.

[29] Berkowitz A.E., Mitchell J.R., Carey M.J.. Young A.P, Zhang S., Spada F.E., Parker F.T Hutten A., et Thomas G., Phys. Rev. Lett. 68 (1992) 3745.

[30] Xiao J.Q., Samuel-Jiang J.S., et Chien C.L., Phys. Rev. Lett. 68 (1992) 3749.

[31] Xiao J.Q., Samuel-Jiang J., et Chien C.L., Phys. Rev. B 46 (1992) 9266.

[32] Parkin S.S.P., Farrow R.F.C., Rabedeau T.A., Marks R.F., Harp G.R., Lam Q., Chappert C Toney M.F., Savoy R., et Geiss R., Europhys. Lett. 22 (1993) 455.

[33] Xiao G., Wang J.Q., et Xiong P., Appl. Phys. Lett. 62 (1993) 420.

[34] Dieny B., Teixeira S.R., Rodmacq B., Cowache C., Auffret S., Redon O., et Pierre J., J. Magr Magn. Mater. 130 (1994) 197. 
[35] Teixeira S.R., Dieny B., Chamberod A., Cowache C., Auffret S., Auric P., Rouvière J.L., Redon O., et Pierre J., J. Phys.: Condens. Matter 6 (1994) 5545.

[36] Chien C.L., Xiao J.Q., et Samuel-Jiang J., J. Appl. Phys. 73 (1993) 5309.

[37] Zhang S. et Levy P.M., J. Appl. Phys. 73 (1993) 5315.

[38] Rabedeau T.A., Toney M.F., Marks R.F., Parkin S.S.P., Farrow R.C.F., et Harp G.R., Phys. Rev. $B 48$ (1993) 16810.

[39] Parker M.R., Barnard J.A., Seale D., et Waknis A., J. Appl. Phys. 73 (1993) 5512.

[40] Morel R., Steren L.B., Barthélémy A., Parent F., et Fert A., Surf. Rev. Lett. (sous presse).

[41] Lee W.Y., Deline V.R., Gorman G., Kellock A., Miller D., Neiman D., Savoy R., Vasquez J., et Beyers R., Mat. Res. Soc. Proc. Symp. 313 (1993) 79.

[42] Thompson S.M., Gregg J.F., Staddon C.R., Daniel D., Dawson S.J., Ounadjela K., Hammann J., Fermon C., Saux G., O'Grady K., Grieves S.J., Coey J.M.D., et Fagan A., Phil. Mag. B 68 (1993) 923; Dieny B., Chamberod A., Cowache C., Genin J.B., Teixeira S.R., Ferrer S., et Barbara B., J. Magn. Magn. Mater. 135 (1994) 191.

[43] Chen L.H., Jin S., Tiefel T.H., et Ramesh R., J. Mater. Res. 9 (1994) 1134.

[44] Hylton T.L., Coffey K.R., Parker M.A., et Howard J.K., Science 261 (1993) 1021.

[45] Loloee R., Schroeder P.A., Pratt W.P., Bass J., et Fert A., Physica B (sous presse).

[46] Holody P., Steren L.B., Morel R., Fert A., Loloee R., et Schroeder P.A., Phys. Rev. B 50 (1994) 12999.

[47] Johnson M., Science 260 (1993) 320.

[48] Prinz G.A., Science 250 (1992) 10. 